\title{
Sur les surpressions qui peuvent résulter du remplissage d'une bâche de turbine par ouverture de sa vanne de garde
}

\section{On possible pressure surges resulting from the opening of the head gate prior to filling the turbine casing}

\author{
PAR M. BONNE'T \\ Ingrinieur aU Service des ETudes ET Recherches Hydraviloues \\ D'Electricité de France
}

English synopsis, p. 580.

Au cours d'essais de réception effectués par ses soins, sur les groupes turbine-alternateur de nombreuses usines hydroélectriques, la Division «Essais Extérieurs » du Service des Etudes et Recherches Hydrauliques d'Electricité de France a eu quelques rares occasions d'observer que des surpressions notables peuvent résulter du remplissage d'une bâche de turbine par ouver- ture de sa vanne de garde. Dans un cas où ce phénomène était particulièrement remarquable, puisque la pression maximum observée dépassait sensiblement celle résultant de la disjonction d'un groupe, il nous a paru intéressant d'analyser le phénomène; la présente note résume les résultats obtenus.

\section{I. - DESCRIPTION SOMMAIRE DE L'INSTALLATION}

L'usine utilise l'eau sous une hauteur de chute brute voisine de $110 \mathrm{~m}$. Ses trois groupes sont à axe horizontal et munis d'orifices compensateurs; les groupes $n^{0 s} 2$ et 3 absorbent un débit

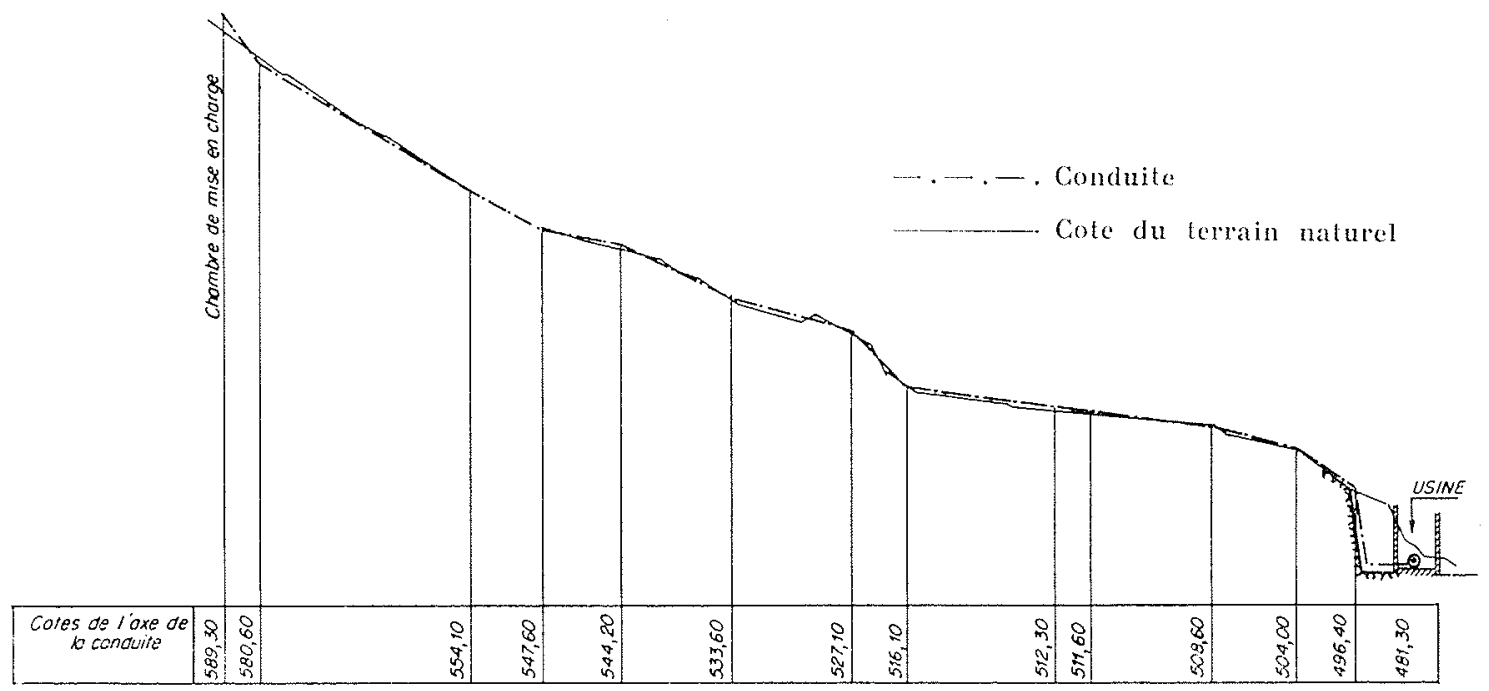

Fig 1. - Profil en long de la conduite. 


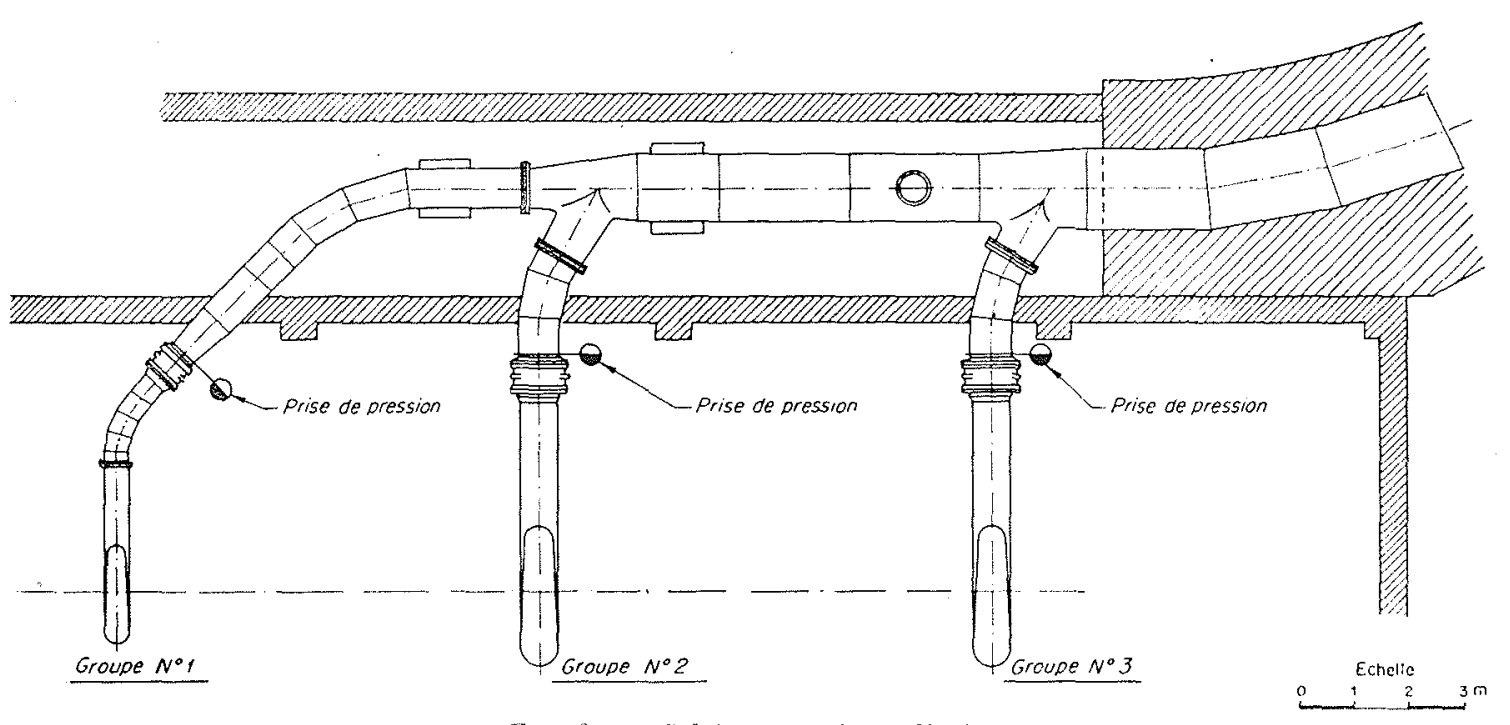

Fia, 2. - Schéma de l'installation

voisin de $3 \mathrm{~m} \% / \mathrm{s}$ et le groupe $\mathrm{n}^{\circ} 1$ un débit de $1 \mathrm{~m}^{3} / \mathrm{s}$ environ; ils sont alimentés à partir d'un canal à écoulement libre, par une conduite forcée de $800 \mathrm{~m}$ de longueur, dont le diamètre varie entre $1,80 \mathrm{~m}$ et $1,50 \mathrm{~m}$.

Les vannes papillon constituant les organes de garde des turbines ne comportent pas de bypass.

La figure $n^{\circ} 1$ donne le profil en long de la conduite forcée; la figure $n^{\circ} 2$ montre le raccordement des groupes au collecteur.

\section{II. - REALISATION DES ESSAIS. - RÉSULTATS OBTENUS}

Tous les essais, sauf un, ont été réalisés dans les mèmes conditions: la vanne de garde a été ouverte après un certain temps d'arrêt du groupe pendant lequel le distributeur était resté fermé; préalablement au premier essai effectuć sur le groupe $n^{\circ} 2$, la bâche avait été partiellement vidangée par ouverture momentanée du distributeur.

Les variations de pression étaient mesurées à $0,50 \mathrm{~m}$ à l'amont de la vanne de garde.

Nous reproduisons dans la figure $n^{\circ} 3$ les enregistrements obtenus. Les courbes relevées ont toutes la mème allure. Après une légère dépression, on observe une surpression de valeur élevée. Par suite des réflexions successives à la chambre d'eau, cette perturbation donne naissance à des oscillations périodiques.

La valeur de la surpression maximum observée à la base de la conduite est très importante : $27 \mathrm{~m}$ pour l'un des essais, à peine inférieure à la valeur garantie en cas de fermeture totale ou partielle $(27,25 \mathrm{~m})$; elle dépasse de beaucoup la valeur de la surpression maximum consécutive à la décharge du groupe $(18 \mathrm{~m})$.

Le tableau $n^{\circ} 1$ ci-contre rassemble les résultats relatifs aux divers essais'.

\section{III. - INTERPRETATION DES RESULTATS D'ESSAI}

L'ouverture de la vanne de garde engendre successivement à la base de la conduite une dépression, due au débit de remplissage de la bâche, et une surpression, provoquée par l'arrêt de ce débit, lorsque la pression de l'air emprisonné dans la bâche atteint la valeur correspondant à la pression statique de la conduite.

Les oscillations de pression qui succèdent à la perturbation initiale sont trés peu amorfies, par suite vraisemblablement de la faible valeur du débit de fuite du distributeur.
Pour suivre l'érolution du phénomène et déterminer la répartition des surpressions le long de la conduite forcée, nous avons utilisé la méthode graphique de Brrgrron.

$$
\text { A. - Etude du phénomène }
$$

par la méthode Brargeron (1).

Les données de construction de l'épure sont,

(1) Nous n'avons pas jugé utile de reproduire ici les diverses épures Bergeron mentionnées à ce paragraphe. 


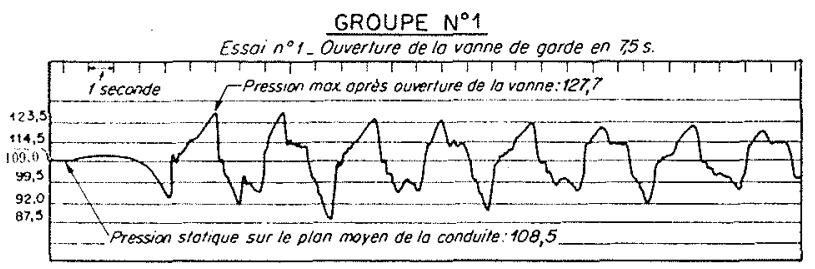

GROUPE $\mathrm{N}^{\circ} 2$

Essor n' ${ }^{\circ}$. Ouverture de lo vanne de garde en 37,5s, opdés vidonge partielle de lo böche por ou-

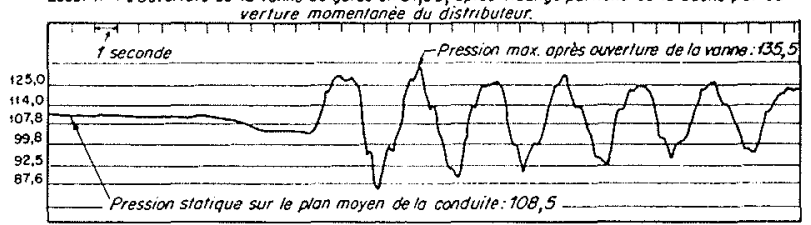

Essor n 2. Ouverlure de lavonne de gorde en $37,5 \mathrm{~s}$.

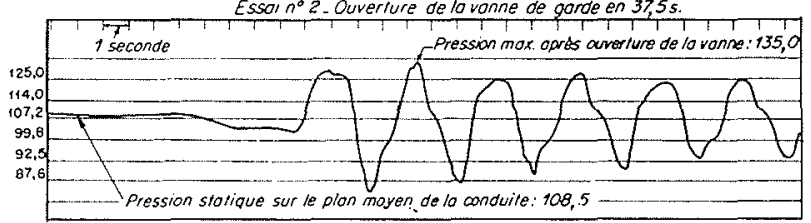

Essain ${ }^{\circ}$ - Quverture de la vanne de garde en $37,5 \mathrm{~s}$.

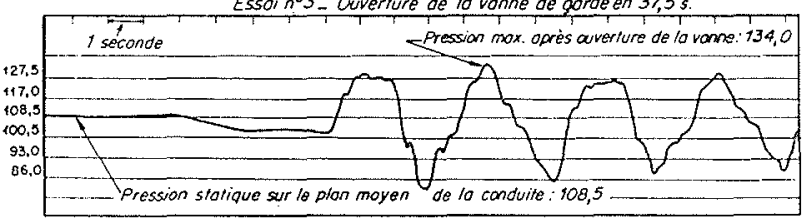

GROUPE $N^{\circ} 3$

Essai n'1, Ouverture de lo vanne de garde en $38,5 \mathrm{~s}$

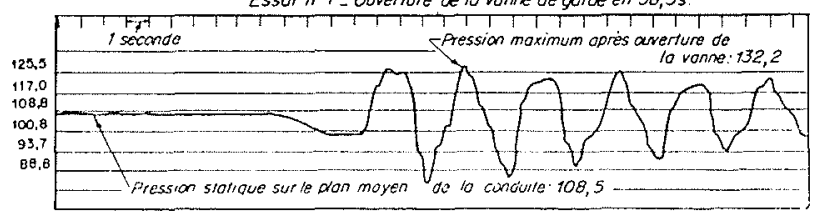

Fid. 3. - Relevés des surpressions a l'amont de la vanne de garde

TableaU $N^{\prime \prime} 1$

RÉsultats I'Essai

\begin{tabular}{|c|c|c|c|c|c|}
\hline \multirow{2}{*}{$\begin{array}{l}N^{\prime \prime} \\
\text { DE L'ESSAI }\end{array}$} & \multirow{2}{*}{ 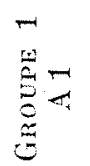 } & \multicolumn{3}{|c|}{ Grovpe 2} & \multirow{2}{*}{ 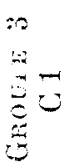 } \\
\hline & & $\vec{\varphi}$ & $\begin{array}{l}\ddot{1} \\
-\infty\end{array}$ & 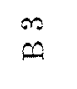 & \\
\hline $\begin{array}{l}\text { Temps d'ouver- } \\
\text { ture de la van- } \\
\text { ne ............ s } \\
\text { Surpression } \\
\text { maximum au- } \\
\text { dessus de la } \\
\text { pression stati- } \\
\text { que .......... }\end{array}$ & 19,2 & 37,5 & 37,5 & 37,5 & 38,5 \\
\hline
\end{tabular}

pour la durée correspondant au temps de remplissage de la bâche, la variation de pression

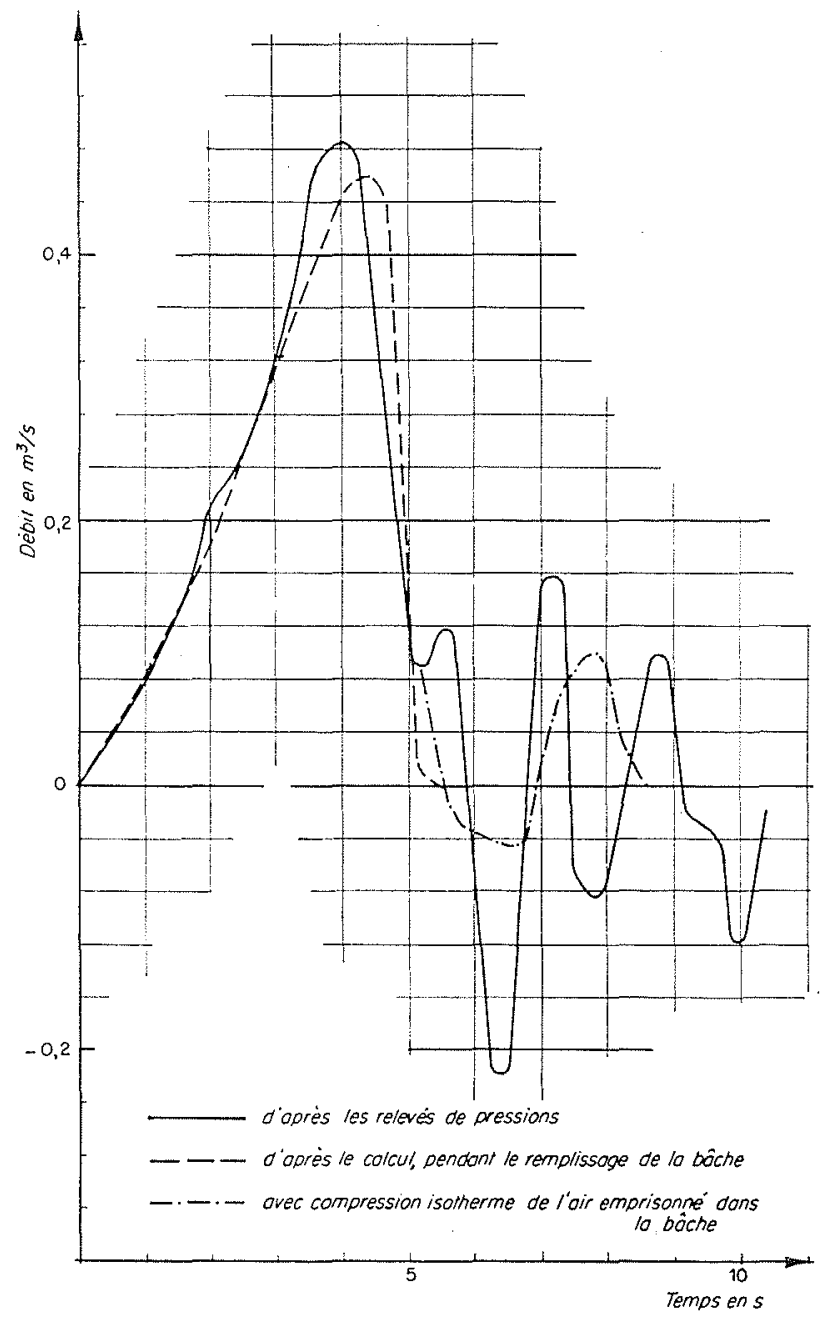

Fra. 4. - Variations du débit à la vamne de garde.

relevée à l'amonl de la ranne de garde au cours de l'essai $n^{\circ} 1$ du groupe $n^{\circ} 2$ et la célérité des ondes.

La loi de variation du débit pendant cette période, telle qu'elle résulte de l'épure, a été représentée sur la figure n" 4 , où a été tracée également la courbe déduite du caleul direct effectué d'après la loi d'ouverture de la vanne et les caracteristiques géométriques de la bâche de la turbine.

Ce calcul a été mené en admettant que le débit traversant la conduile est à chaque instant proportionnel à la section $\mathrm{S}$ laissée libre par la vanne, corrigée d'un coefficient de contraction pris égal à 0,8 et à la racine carrée de la différence des pressions $\Delta$ h existant entre l'amont et l'aval de cette vanne:

$$
\mathrm{Q}=\mathrm{S} \times 0,8 \times \sqrt{2 \mathrm{g \Delta h}}
$$

Au début de la manœuvre d'ouverture, la 
contre-pression au distributeur est tres voisine de la pression atmosphérique, jusqu'à ce que le plan d'eau s'établisse au niveau du point le plus haut du distributeur. Ensuite, l'air emprisonné sous la culotte supérieure de la bâche crée une contre-pression calculée d'après la loi de MARIOTTE.

On peut constater que la courbe obtenue en admettant cue l'ouverture de la vanne s'effectue à vitesse angulaire constante pendant les premiers instants de son mouvement, est très voisine de celle résultant de l'épure Bragros (1). Celle-ci doit done représenter assez fidèlement les phénomènes réels pendant la durée de remplissage de la bâche.

Pour la période postérieure, nous avons tracé une épure utilisant encore comme donnée la variation de pression observée.

Elle conduit, par suite des irrégularités de l'enregistrement des pressions, à une loi de débit à la vanne de garde qui semble assez arbitraire, et que nous avons représentée sur la figure n" 4 .

(1) Ajoutons que le volume de la bâche, calculé d'après la loi de variation de débit déduite de l'épure, est de $1,3 \mathrm{~m}^{3}$. valeur pratiquement égale au volume eflectif.
Par suite, il nous a paru prudent d'évaluer l'influence d'une modification de la loi de débit à la vanne de garde sur la loi de répartition des surpressions le long de la conduite.

Nous avons établi dans ce but deux épures : - Dans l'une, le débit est supposé constamment nul à la vanne de garde,

- Dans l'autre, nous avons admis une compression isotherme de l'air emprisonné à la partie supérieure de la bâche.

La courbe de variation de débit à la vanne de garde qui s'en déduit est représentée sur la figure $\mathrm{n}^{\circ} 4$ précitée.

La figure n" 5 reproduit, outre l'enregistrement relevé, les variations de pression à la vanne de garde d'après ees deux épures. Elle met en évidence l'influence de la poche d'air, qui tend à accroitre l'amplitude des surpressions et des dépressions.

La courbe relevée se place entre celles qui correspondent respectivement à l'absence de poche d'air, et à une variation de pression isotherme de l'air comprimé dans la bâche.

La comparaison des résultats des trois épures montre que la répartition des surpressions le

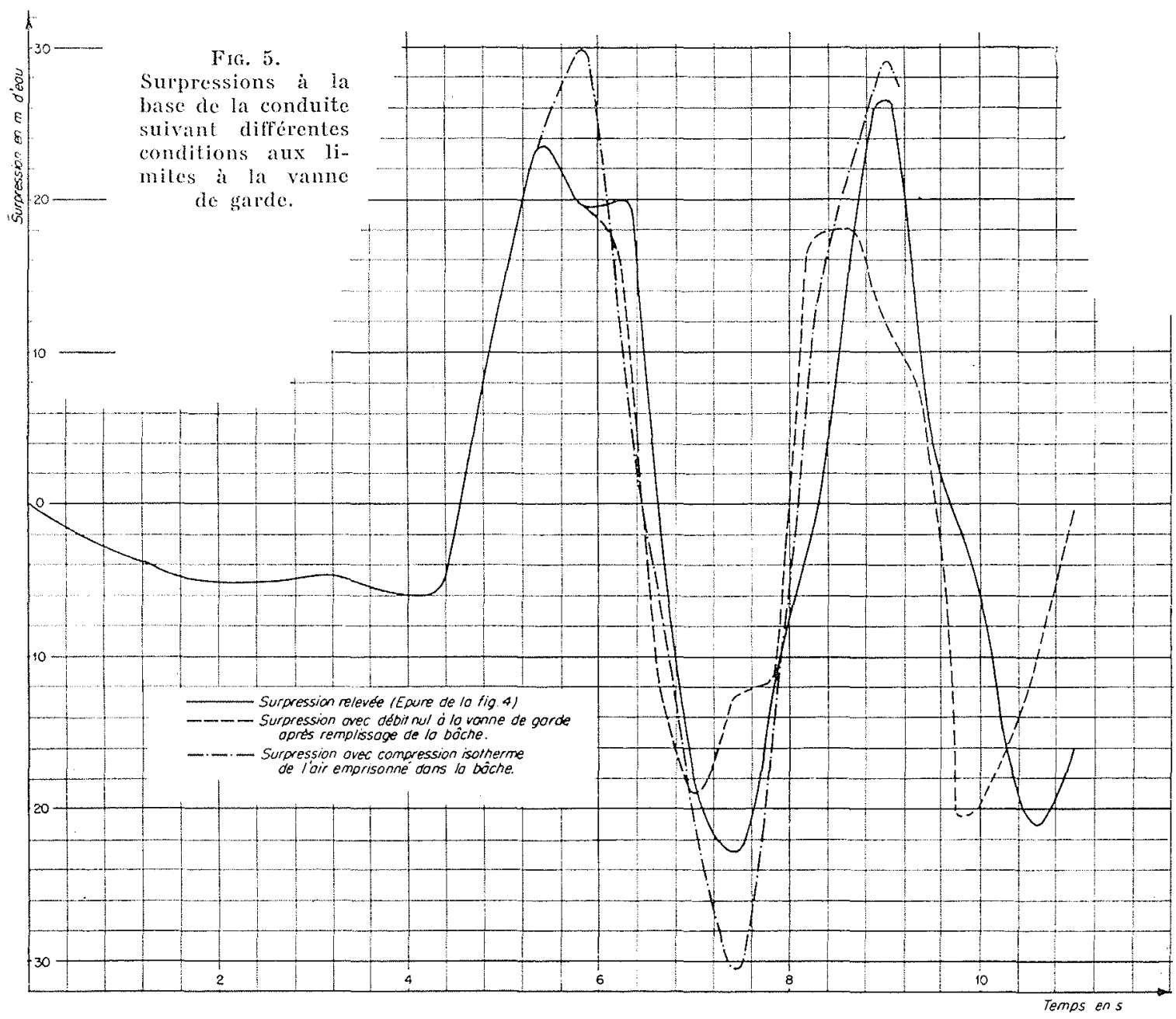




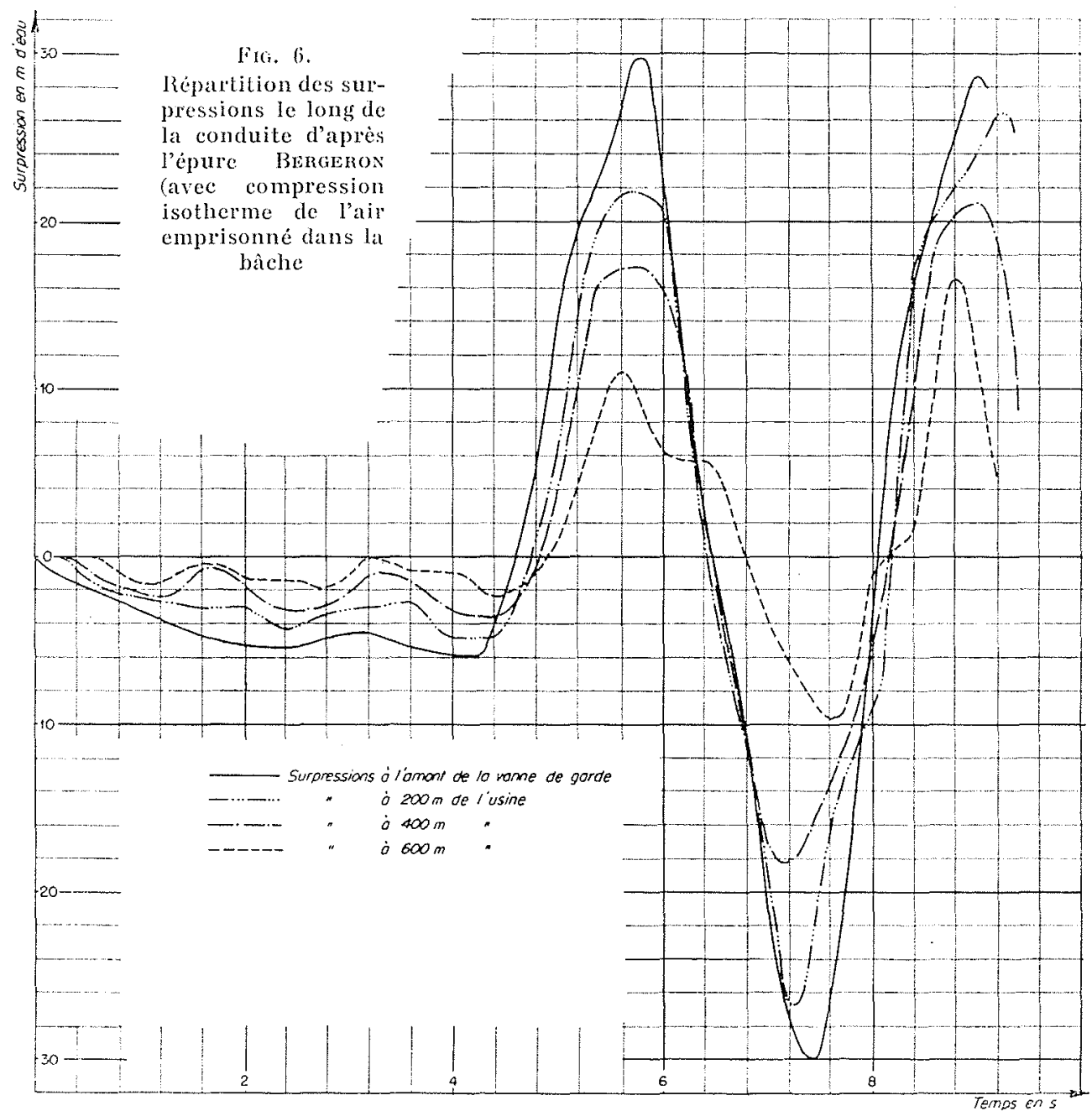

long de la conduite est toujours approximativement la même, ainsi qu'en témoigne le tableau ci-après :

$$
\text { TABLEAU } \mathrm{N}^{\circ} 2
$$

RÉPARTTTION DES SURPRESSIONS LE LONG DE LA CONDUITE POUR DIFFERENTES CONDITIONS AUX LIMITES AVAL.

\begin{tabular}{|c|c|c|c|c|}
\hline \multirow{2}{*}{$\begin{array}{l}\text { CONDTTIONS AUX LIMITYS } \\
\text { AVAL DE L'ÉPURE }\end{array}$} & \multicolumn{4}{|c|}{$\begin{array}{c}\text { SURPRESSIONS MAXIMA } \\
\text { AE-DESSUS IOE LA PRESSION } \\
\text { STATTQUE }\end{array}$} \\
\hline & 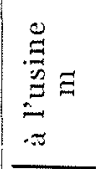 & 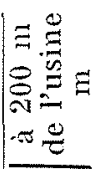 & 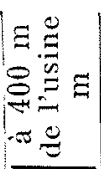 & 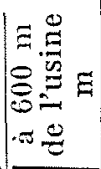 \\
\hline Surpression relevée & 27 & 23,5 & 17,5 & 11 \\
\hline Débit nul.... & 23,5 & 22 & 17 & 13 \\
\hline $\begin{array}{l}\text { Compression isotherme } \\
\text { de l'air dans la bâche. }\end{array}$ & 30 & 26,5 & 21 & 16,5 \\
\hline
\end{tabular}

\section{B. - Répartition des surpressions LE LONG DE LA CONDUITE}

Nous avons représenté, à titre d'exemple, sur la figure $\mathrm{n}^{\circ} 6$, les surpressions en quatre points distants de $200 \mathrm{~m}$, en admettant une compression isotherme de la poche d'air.

La figure $n^{\circ} 7$ reproduit la courbe enveloppe des pressions maxima le long de la conduite (1). On peut constater que la valeur de la surpression diminue relativement peu quand on s'éloigne vers l'amont.

La coupure du débit maximum de remplissage de la bâche intervenant à partir d'un régime permanent, et suivant la Ioi de décroissance déduite de l'épure, engendrerait un coup de bélier dont la valeur maximum serait voisine de $21 \mathrm{~m}$ et dont la répartition le long de la con-

(1) La répartition des surpressions représentée est la plus défarorable de celles qui résultent des tracés des trois épures. 


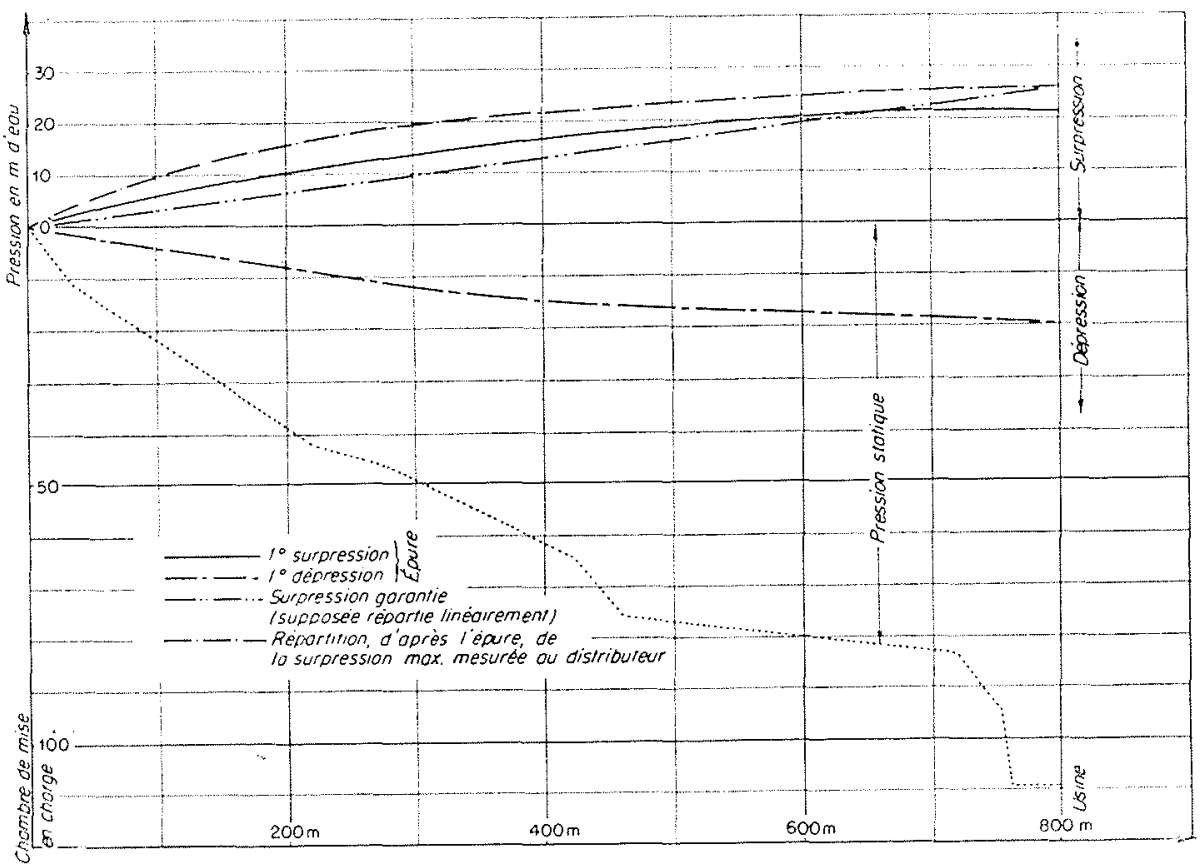

Fug. 7. -- Répartition des pressions le long de la conduite.

duite s'écartcait moins de la répartition linéaire que la surpression obtenue (2).

L'excédent de pression observé dans le cas actuel sur la majeure partie de la conduite est dû à l'augmentation du débit précédant l'arrêt; en effet, les ondes de dépression dues à cette variation de débit se réfléchissent avec changement de signe à la chambre d'eau, et interfèrent avec les ondes de surpression créés par la décroissance du débit.

(2) Il est a remarquer que le débit s'annule en $1,6 \mathrm{~s}$ environ, durée qui caractérise la limite entre les fermeture lentes et rapides.
La superposition de ces deux ondes positives peut amplifier, en certains points, la valeur de la pression.

Il est donc raisonnable d'admettre que, dans une grande partie de la conduite, la valeur absolue maximum de la surpression peut être sensiblement la même qu'au distributeur. En admetlant que les conditions réalisées au cours de l'essai $n^{\circ} 1$ du groupe $n^{\circ} 2$ soient les plus défavorables, sa valeur peut dépasser, dans la partie amont de la conduite, $50 \%$ de la pression statique, ainsi que le montre le tableau ci-dessous.

Tableau $\mathrm{N}^{\circ} 3$

REPARTITION DES SURPRESSIONS

LE LONG DE LA CONDUITE

\begin{tabular}{|c|c|c|c|c|c|c|c|c|c|c|}
\hline $\begin{array}{c}\text { DISTANCE DU PONN CONSIDÉRÉ } \\
\text { A L'USINE }\end{array}$ & 0 & 100 & 200 & 300 & 400 & 500 & 600 & 650 & 700 & 800 \\
\hline Surpression absolue............... m & 27 & 27 & 26 & 23 & 20 & 19 & 16 & 14 & 13 & 0 \\
\hline Pression statique................ & 109 & 86 & 81 & 76 & 62 & 49 & 39 & 30 & 23 & 0 \\
\hline $\begin{array}{l}\text { Surpression relative rapportée à la pres- } \\
\text { sion statique.................... }\end{array}$ & 24 & 32 & 32 & 30 & 32 & 39 & 41 & 48 & 57 & 0 \\
\hline
\end{tabular}




\section{CONCLUSION}

Cet exemple montre que le remplissage de la bâche d'une turbine (dont le distributeur est fermé et relativement étanche) par ouverture de sa vanne de garde peut, dans certains cas particuliers, produire une surpression excédant celle correspondant à la décharge brusque du groupe; le danger de cette surpression est accru par le fait que sa répartition le long de la conduite n'est pas linéaire mais peut se rapprocher de la répartition dite « intégrale » produite par un coup de bélier de «fermeture rapide». Ce phénomène est à craindre notamment dans le cas d'une conduite d'assez grande longueur et d'une vanne de garde ne comportant pas de by-pass.

Les calculs précédents montrent qu'il est possible d'évaluer dans chaque cas la valeur des surpressions à craindre en tous points de la conduite.

Il semble d'ailleurs facile de réduire l'importance de ces surpressions : il suffit d'assurer le remplissage de la bâche au moyen d'un débit suffisamment faible pour que les dépressions initiales et les surpressions qui leur succèdent aient une valeur très réduite; en l'absence d'un by-pass, cela peut ètre obtenu en effectuant le mouvement d'ouverture en deux étapes, la première étant constituée par un simple décollage de la vanne, suivi d'un temps d'arrêt dont la durée serait déterminée expérimentalement, mais qui, dans le cas analysé ici, n'excéderait vraisemblablement pas une vingtaine de secondes.

\section{I S C U S I O N}

M. le Président remercie M. Bonver et demande si, dans le cas où l'air est emprisonné sous la calotte supérieure de la bàche l'écart entre la courbe réclle et la courbe déduite de l'application de la loi de Manotéte ne viendrat pas de ce qu'on serait plus près d'une compression adiabatique que d'une compression isotherme.

M. Bonnet estime que cette explication est plausible, mais n'a pas essayé de la vérifier. Il signale une autre. raison possible de la discordance de ces deux courbes: c'est le fait que la vitesse angulaire d'ouverture de la ranne n'est peut-être pas linéaire pendant le premicr. instant de fonctionnement, ainsi que cela a été supposé, car le couple a fournir diminue au fur et à mesure que la contre-pression s'accroît.

M. Gaben compare les experiences de M. Bonnet oil le distributeur était bien fermé et lamortissement de la surpression très lent, avec les essais pratiques de réception de turbine de MM. REMÉvínas el Bourgulinon, menfionnés a propos de la communication précédente, dans lesquels, la turbine restant en ouverture de marche a vide, c'est-à-dire incomplétement ferméc, la moindre ouverture pouvait ctre une cause des amortissements rapides des coups de bélier constatés.

M. REMÉninas est bien d'accord sul l'importance des fuites sur l'amortissement des coups de bélier. Il precise que, dans l'expérience relatée par M. Bonver, la bâche de la turbine est déjà remplie d'eau plus qu'à moitić; donc le volume à remplir sous le débit de la vanne est relativement faible, ce qui explique la mpidité de la coupure de ce débit.

Au nom de M. Escanos, empêché d'assister à la séance, M. RÉmÉNLÉRAS résume une observation faite par ce dernier dans une usine des Pyrénées. Celle-ci comportait une galerie d'amenée en charge, une chambre d'écuilibre a etranglement et une conduite force alimentant sous une chute de 800 mètres des turbines Pelton. A u moment ot les essais de réception allaient être cffectués une manouvre intempestive de la vanne de garde de la turbine, à l'arrêt, a provoqué un violent coup de bélicr à front raide et engendré quelques fissures dans le canal d'amenée, au voisinage de la chambre d'éguilibre. Le phénomène est tout à fait analogue à ceux dont M. BONNET a parlé dans sa communication. Si l'on fermo d'abord la vanne de garde et ensuite l'injecteur d'une turbine Pelton en service, l'intervalle entre ecs deux organes se trouve rempli d'air; si à ce moment on ouvre la vanne de gatede, ec volume d'air, très faible, se comprime rapidement à la pression correspondant a la chute statique de $800 \mathrm{~m}$ et le débit de la vanne de garde se trouve coupe en un temps tres cont. M. Tscanis a mesuré dans ces conditions, une surpression de $232 \mathrm{~m}$ inmédiatement à l'amont de la vanne de garde. Lat transmission de cette surpression, le long de la conduite élant ici encore « quasi intégrale » il en résulte des surpressions au voisinage de la cheminée d'équilibre qui expliquent fort bien les fissures constatees dans cette région.

M. Gaben dit que ce phénomene, dangercux surtout dans les turbines Pelton, est bien connu des turbinies qui y parent sans peine en préroyant des by-pass sur les vannes de garde et en manouvant ces by-pass suivant une consigne convenable comme le proposcut MII. RËménétras et Bonnet.

M. Réméñéras ajoute que certaines observalions faites au cours d'essais de réception montrent que les cas cités respectivement par M. BONNeT et par M. Escande ne sont certainement pas isolés. 